\title{
Adiabatic Cooling of Atoms by an Intense Standing Wave
}

\author{
Jian Chen, J. G. Story, J. J. Tollett, and Randall G. Hulet \\ Department of Physics and Rice Quantum Institute, Rice University, Houston, Texas 7725I-1892
}

(Received 14 May 1992)

\begin{abstract}
Lithium atoms channeled in the nodes of an intense standing-wave radiation field are cooled to near the recoil limit by adiabatically reducing the radiation intensity. The final momentum distribution has a narrow component with a root-mean-squared momentum of $2 \hbar k$ in one dimension, where $\hbar k$ is the momentum of a radiation-field photon. The data are compared with the results of a Monte Carlo simulation using a two-level atom model. This process may be useful for cooling and increasing the phasespace density of atoms confined in a magnetic trap.
\end{abstract}

PACS numbers: $32.80 . \mathrm{Pj}, 42.50 . \mathrm{Vk}$

Recent laser cooling experiments have demonstrated that the momentum of an atom can be reduced to near that of a single photon of the laser radiation field, $\hbar k$ $[1,2]$. The corresponding kinetic temperature of atoms cooled to this "recoil limit" is approximately $1-10 \mu \mathrm{K}$. The cooling mechanism in these experiments was found to rely on optical pumping among the degenerate ground states of the atom [3]. In this paper, we report cooling atoms to near the recoil limit in one dimension, using a method that requires only two atomic levels.

An atom moving in a near-resonant standing-wave radiation field experiences a periodic, spatially varying potential energy due to the interaction of the induced electric dipole moment of the atom with the field. The atom can experience a force in the standing wave since the gradient of the potential energy may be nonzero. For a radiation field with frequency blue-detuned from resonance (i.e., frequency greater than the atomic resonance frequency), the atoms will be attracted to the nodes of the standing wave. In this case, atoms whose maximum kinetic energy is less than the depth of the potential may be trapped around the nodes [4]. This "channeling" of atoms has been observed [5]. Once the atoms are channeled, their kinetic energy may be reduced by adiabatically lowering the standing-wave intensity. Adiabatic cooling of channeled atoms has been discussed in the context of low-intensity "optical molasses" [2,6]. In our experiment, atoms channeled in an intense, one-dimensional standing wave are cooled adiabatically to nearly the recoil limit.

In the absence of spontaneous emission, a two-level atom in an intense, near-resonant standing wave possesses two states with different effective potentials, corresponding to the sign of the atom's induced dipole moment. These potentials are $U \pm(z)= \pm \frac{1}{2} \hbar\left[\Omega_{0}^{2} \sin ^{2}(k z)+\Delta^{2}\right]^{1 / 2}$ $-\frac{1}{2} \hbar \Delta$, where $\Omega_{0}$ is the on-resonance Rabi frequency (i.e., $\hbar \Omega_{0}$ is the interaction energy) at an antinode of the standing wave, $\Delta$ is the detuning of the standing-wave frequency from resonance, and $k$ is the wave vector of the radiation which is along the $z$ axis [7]. When the field energy is included, these effective potentials correspond to the two "dressed state" eigenvalues [8]. $U_{ \pm}(z)$ may be adequately approximated by a harmonic function for atoms whose oscillation amplitude is small compared with the optical wavelength. For example, consider the $2 S-2 P$ transition of the ${ }^{7} \mathrm{Li}$ atom. The wavelength of this transition is $\lambda=671 \mathrm{~nm}$ and the spontaneous decay rate of the $2 P$ state is $\gamma=(2 \pi) 5.8 \mathrm{MHz}$. For an atom channeled in the quantum-mechanical ground state of $U_{+}$with $\Omega_{0}$ $=45 \gamma$ and $\Delta=13 \gamma$, the oscillation amplitude is $0.02 \lambda$. In this case, the oscillation frequency is $8 \mathrm{MHz}$, and the root-mean-squared (rms) atomic momentum is $5.5 \hbar k$. If the potential is reduced by lowering the standing-wave intensity (i.e., $\Omega_{0}$ ) at a rate that is slow in comparison with the atom's oscillation period, the atom's kinetic energy will be reduced. When $\Delta=13 \gamma$ and $\Omega_{0}$ is reduced to $\sim \gamma$, the potential just supports a single bound state with a rms momentum of $\sim \hbar k$. This limiting momentum approximately corresponds to the one given by the uncertainty principle for an atom localized to $\Delta z \sim \lambda / 2$.

In the experiment, shown schematically by Fig. 1, a collimated, thermal beam of lithium atoms is crossed at $90^{\circ}$ by an intense, blue-detuned standing wave. The

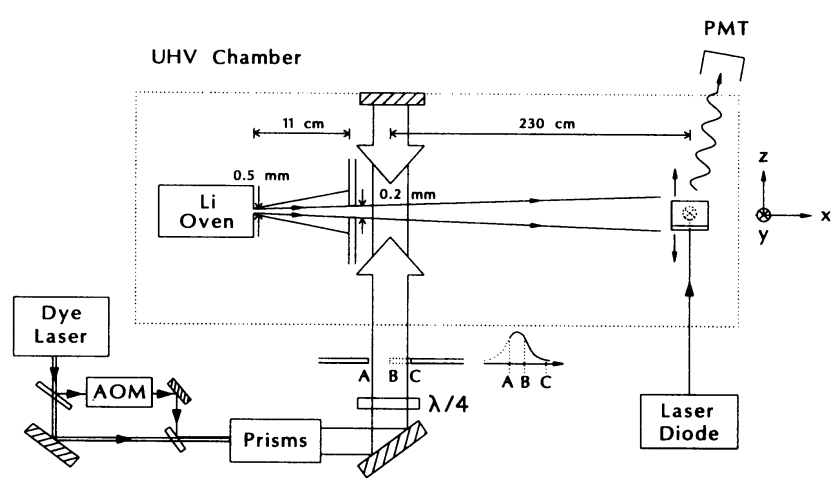

FIG. 1. Schematic diagram of the apparatus. AOM, acousto-optic modulator; prisms, four beam expanding prisms; $\lambda / 4$, quarter-wave plate; PMT, photomultiplier. Points $A, B$, and $C$ correspond to the positions of the standing-wave aperture. Aperture at point $A$ on the leading edge is fixed, while the trailing edge of the standing wave is apertured either at point $B$ or $C$. 
atoms pass through the standing wave, which has a Gaussian intensity distribution along the atomic beam ( $x$ axis). In the intense region of the standing wave the transverse velocity of the atoms is reduced by the dissipative dipole force $[8,9]$. This interaction causes many of the atoms to be channeled in the nodes. As the atoms pass through the Gaussian tail of the standing wave they experience a slowly decreasing intensity. If the decrease in intensity is sufficiently slow, the atoms will be adiabatically cooled. In this experiment, adiabaticity requires that the time for an atom to pass through the Gaussian waist of the standing wave be much larger than the oscillation period of a channeled atom.

The standing wave is produced by retroreflecting a laser beam from a frequency-stabilized dye laser using a mirror located inside the vacuum chamber. The laser beam is collimated and expanded to a Gaussian waist ( $e^{-2}$ point of maximum intensity) of $9.2 \mathrm{~mm}$ along the atomic beam axis ( $x$ axis), and to $1.4 \mathrm{~mm}$ along the $y$ axis. A quarter-wave plate is used to produce nearly circular polarization. A quantization axis is defined by the standing wave and a collinear magnetic field of $8 \mathrm{G}$ produced by a Helmholtz coil pair. The interaction region is shielded from ambient magnetic fields using a high- $\mu$ metal tube aligned collinear with the applied magnetic field. The laser frequency is offset locked using saturated absorption spectroscopy in a heat pipe from the $2 S_{1 / 2}$, $F=2 \leftrightarrow 2 P_{3 / 2}, F=3$ resonance frequency of the ${ }^{7} \mathrm{Li}$ atom, so that $\Delta=13 \gamma$. The laser power is $520 \mathrm{~mW}$, giving a peak intensity in the standing wave of $10.3 \mathrm{~W} / \mathrm{cm}^{2}$. The saturation intensity (i.e., $\Omega_{0}=\gamma$ ) of the $2 S_{1 / 2}, F=2$ $\leftrightarrow 2 P_{3 / 2}, F=3$ transition using circularly polarized light is $5.1 \mathrm{~mW} / \mathrm{cm}^{2}$, yielding $\Omega_{0}=45 \gamma$. A second laser beam, frequency shifted with an acousto-optic modulator from the standing-wave frequency, is used to prepump atoms initially in the $F=1$ ground state into the $F=2$ state (ground-state hyperfine splitting of $803 \mathrm{MHz}$ ), and to repump atoms which fall out of the $2 S_{1 / 2}, F$ $=2 \leftrightarrow 2 P_{3 / 2}, F=3$ cycling transition. The intensity of this beam is relatively low, $-15 \mathrm{~mW} / \mathrm{cm}^{2}$, and it is retroreflected at an angle so that it does not form a standing wave. The optical quality of all the optical components was carefully controlled to minimize the number of defects in the standing wave that could interfere with channeling.

The effect of the standing wave on the transverse velocities in the beam is probed downstream of the interaction region using a focused beam from a diode laser [10]. The probe beam is parallel to the $x-y$ plane and intersects the atomic beam nearly orthogonally, at an angle of $\sim 88^{\circ}$ relative to the atomic beam axis. The probe laser, whose frequency is offset locked from the $2 S_{1 / 2}, F=2 \leftrightarrow 2 P_{3 / 2}$, $F=3$ resonance frequency by $45 \mathrm{MHz}$, will preferentially excite atoms with a longitudinal velocity centered around $900 \mathrm{~m} / \mathrm{s}$, with a Lorentzian width of $\sim 150 \mathrm{~m} / \mathrm{s}$ due to the linewidth of the transition. Since the most probable velocity in the atomic beam is $\sim 1700 \mathrm{~m} / \mathrm{s}$, only the slow velocity tail of the distribution is probed. The use of this slower velocity both ensures the adiabaticity of the process, and increases the fraction of atoms channeled in the high-intensity region. The atomic fluorescence induced by the probe beam is collected by a lens, detected by a photomultiplier, and individual photons are counted and recorded by a computer. The position of the focused probe beam is scanned in the $z$ direction, across the atomic beam, using a mirror mounted on a translation stage to record the transverse distribution of the atoms in the beam. For a single longitudinal velocity, there is a oneto-one correspondence between transverse position and transverse velocity. The Gaussian beam waist of the focused probe beam of $50 \mu \mathrm{m}$ together with the dimension of the collimating slit yield a transverse velocity resolution of $8 \mathrm{~cm} / \mathrm{s}$, or a momentum resolution of better than $1 \hbar k$. The spread of longitudinal velocities contributing to the signal broadens the transverse momentum resolution for transverse momenta greater than $6 \hbar k$.

Figure 2 shows experimental data as bold lines, while the lighter lines are the results of a Monte Carlo simulation described below. Figure 2(a) shows the initial transverse momentum distribution when no standing wave is

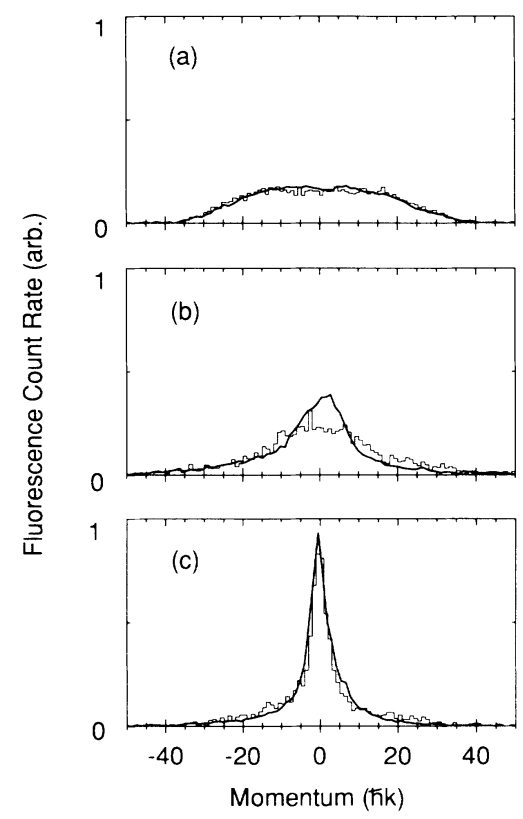

FIG. 2. Data (bold lines) and results of the Monte Carlo simulation (light lines) for the transverse momentum distribution of atoms in the beam. (a) No standing wave present, corresponding to the original distribution. (b) Standing wave apertured at points $A$ and $B$ of Fig. 1, so that the atoms interact only with the most intense $1-\mathrm{cm}$ portion of the beam. Atoms are channeled during this interaction but no significant adiabatic cooling is possible in this case. (c) Standing wave apertured at points $A$ and $C$ of Fig. 1, so that atoms interact with the decreasing intensity tail of the standing wave. In this case, an adiabatic lowering of the intensity cools the atomic motion along the standing wave, producing an rms momentum of $2 \hbar k$. 
present. Figure 2(b) corresponds to the case where the standing wave is apertured, as shown by points $A$ and $B$ in Fig. 1, so that the atoms can interact only with the most intense $1-\mathrm{cm}$ length of the standing wave. In the apertured region, $\Omega_{0}$ varies from $33 \gamma$ at the edges of the aperture to its peak value of $45 \gamma$ at the center of the standing wave. In this high-intensity regime, the resulting momentum distribution is determined by the balance of the dissipative cooling due to the time-averaged dipole force and the diffusional heating due to the fluctuations of this force $[8,9,11,12]$. Although no significant adiabatic cooling is possible due to the small change of Rabi frequency, a large fraction of the atoms are channeled in this region. The half width at half maximum of the momentum distribution is $8 \hbar k$, which is comparable to the rms momentum of $5 \hbar k$ for an atom channeled in the ground state of the potential with $\Omega_{0}=33 \gamma$. Finally, for the data shown in Fig. 2(c), the aperture was moved out to point $C$, as indicated in Fig. 1, so that the atoms could experience the Gaussian tail of the standing wave. At point $C$, the intensity of the standing wave has decreased to the level where $\Omega_{0} \approx 0.4 \gamma$. This case corresponds to the adiabatic reduction of the standing-wave intensity, so that channeled atoms will be adiabatically cooled. The exact position of point $C$ had little effect on the final distribution. Although a blue-detuned standing wave is heating at low intensities, the force on the slow atoms of interest here with Doppler shifts $k v \ll \Delta$ is very weak. The data of Fig. 2(c) can be fitted well by two Gaussian functions, each with $50 \%$ of the total number of atoms, one with a rms momentum of $2 \hbar k$ and the other with a rms momentum of $10 \hbar k$. In the simulations described below the atoms which make up the narrow component were deeply channeled while the others were either unchanneled or channeled with high energy. The rms momentum of the narrow component is a factor of 2 above the width expected for atoms cooled to the recoil limit, and a factor of 2.4 below that for atoms cooled to the "Doppler limit" [1].

This adiabatic cooling process requires only two atomic levels, a ground and an excited state. An effective twolevel cycling transition is formed by circularly polarizing the radiation so that atoms in the $F=2, m_{F}=2$ ground state can be excited only to the $F=3, m_{F}=3$ excited state. However, for the data shown in Figs. 2(b) and 2 (c), the quarter-wave plate crystal axis was at an angle of $32^{\circ}$ with respect to the polarization direction of the incident linearly polarized light, rather than at $45^{\circ}$, as required to produce circular polarization. Therefore, the standing-wave radiation was slightly elliptically polarized. We found that this polarization produced the narrowest final momentum distribution. Additional experiments established that there is a three or more level cooling process involving the $F=1$ ground state, which is more efficient than the usual two-level dissipative dipole force for cooling and channeling atoms in an intense, blue-detuned standing wave [13]. "Sisyphus" cooling schemes involving two atomic levels coupled by a nearresonant standing wave, ard a third, "reservoir" level have recently been proposed in the literature [14]. We emphasize that this multilevel scheme is an efficient way to "load" atoms into the channels, but the adiabatic cooling involving only two atomic levels produces the striking cooling evident in Fig. 2(c). We were able to exclude the possibility that our data could be explained by any cooling mechanism involving multiple ground-state $m_{F}$ levels in two ways. First, we excluded the repumping beam from the trailing edge (point $B$ to $C$ ) of the standing wave and measured the resulting momentum distribution. The elliptically polarized standing wave couples the $F=2$ ground state to the nearly degenerate $F=1,2$, and 3 excited states. Therefore, if the atoms were cooled by a mechanism involving spontaneous decay into ground-state sublevels other than $F=2, m_{F}=2$, the atoms would be quickly optically pumped into the $F=1$ ground state because of the large branching ratios for spontaneous decay into the $F=1$ ground state. However, we found that after passing through the standing wave, $50 \%$ of the atoms remained in the $F=2$ ground state, and furthermore, these atoms were left with a momentum distribution having the same shape as that of Fig. 2(c). Second, we attempted to cool atoms using the repumping beam and an elliptically polarized standing wave of uniform, rather than decreasing intensity. We found that narrow features of the sort shown in Fig. 2(c) could not be duplicated with the large range of detunings and Rabi frequencies we investigated. Since the integral of the three signals shown in Fig. 2 is the same to within our experimental resolution, we conclude that few atoms are left in the $F=1$ ground state when the repumping beam is present.

The lighter lines shown in Fig. 2 are the results of a Monte Carlo simulation of the experiment. In this model, an atom is assumed to be in one dressed state. The atom's motion is determined by the instantaneous force given by its potential gradient in the standing wave. Fluctuations in this force come about by spontaneous emission events that cause the atom to change dressed state. Trajectories of 2000 atoms were accumulated to construct the distributions shown in Fig. 2. This model, originally developed by Dalibard et al. [15], was adapted by us to successfully model the evolution of atomic momentum in an intense standing wave [12]. For the results presented in Fig. 2, a two-level system was assumed. Experimentally, the data obtained with circular polarization and the aperture at point $B$ agree very well with the two-level model, but the narrow peak of Fig. 2(c) is not observed when the aperture is moved to point $C$. A possible explanation for this discrepancy is that defects in the standing wave cause atoms to become unchanneled and these atoms are not channeled again sufficiently rapidly to be adiabatically cooled. However, the increased damping provided by the three-level cooling mechanism may channel the atoms more deeply and sufficiently rapidly to allow adiabatic cooling, even with the existence of some 
defects.

Because this adiabatic cooling process involves only two atomic levels, it may be useful for cooling magnetically trapped atoms, for which a two-level system may be isolated by their Zeeman shifts. The combination of the dissipative dipole force and adiabatic cooling by an intense standing wave can result in an increase of phasespace density in the magnetic trap. This can be understood by considering a sample of atoms confined to a region much larger than an optical wavelength by a potential that has a flat bottom, and infinitely steep walls. After the application of the standing wave the atoms can be periodically localized in the optical potential by the dissipation provided by the dipole force. During the adiabatic cooling cycle phase-space density is conserved, but the localization in the optical potential wells is exchanged for a reduction in energy, thereby leaving the atoms confined by the original magnetic potential, but at a lower energy.

We are grateful to N. W. M. Ritchie and C. C. Bradley for their contributions to this work. We benefited by a stimulating discussion with $D$. Kleppner. This work was partially supported by the Texas Advanced Technology Program, the Robert A. Welch Foundation, and the National Science Foundation. R. G. H. is an Alfred P. Sloan Research Fellow and a Shell Faculty Fellow.

[1] P. D. Lett et al., Phys. Rev. Lett. 61, 169 (1988); A. Aspect et al., Phys. Rev. Lett. 61, 826 (1988); C. Salomon et al., Europhys. Lett. 12, 683 (1990); S.-Q. Shang et al.,
Phys. Rev. Lett. 67, 1094 (1991); A. M. Steane and C. J. Foot, Europhys. Lett. 14, 231 (1991).

[2] Y. Shevy, D. S. Weiss, and S. Chu, in Spin Polarized Quantum Systems, edited by S. Stringari (World Scientific, Teaneck, NJ, 1989), pp. 287-294.

[3] J. Dalibard and C. Cohen-Tannoudji, J. Opt. Soc. Am. B 6, 2023 (1989); P. J. Ungar et al., J. Opt. Soc. Am. B 6, 2058 (1989).

[4] V. S. Letokhov, Pis'ma Zh. Eksp. Teor. Fiz. 7, 348 (1968) [Sov. Phys. JETP Lett. 7, 272 (1968)].

[5] M. G. Prentiss and S. Ezekiel, Phys. Rev. Lett. 56, 46 (1986); C. Salomon et al., Phys. Rev. Lett. 59, 1659 (1987); V. I. Balykin et al., Opt. Lett. 13, 958 (1988).

[6] P. D. Lett et al., J. Opt. Soc. Am. B 6, 2084 (1989).

[7] A. P. Kazantsev, Usp. Fiz. Nauk 124, 113 (1978) [Sov. Phys. Usp. 21, 58 (1978)].

[8] J. Dalibard and C. Cohen-Tannoudji, J. Opt. Soc. Am. B 2, 1707 (1985).

[9] J. P. Gordon and A. Ashkin, Phys. Rev. A 21, 1606 (1980); A. P. Kazantsev et al., J. Opt. Soc. Am. B 2 , 1731 (1985).

[10] V. I. Balykin et al., J. Opt. Soc. Am. B 2, 1776 (1985).

[11] A. Aspect et al., Phys. Rev. Lett. 57, 1688 (1986).

[12] J. Chen, J. G. Story, and R. G. Hulet (to be published).

[13] The results of this experiment will be presented elsewhere.

[14] D. J. Wineland, J. Dalibard, and C. Cohen-Tannoudji, J. Opt. Soc. Am. B 9, 32 (1992); D. E. Pritchard and W. Ketterle, in Proceedings of the Varenna Summer School on Laser Manipulation of Atoms and Ions, edited by E. Arimondo and W. P. Phillips (North-Holland, Amsterdam, to be published).

[15] J. Dalibard et al., in Fundamentals of Quantum Optics II, edited by F. Ehlotzky (Springer-Verlag, Berlin, 1987), p. 196. 